Aksaray University
Journal of Science and Engineering
e-ISSN: 2587-1277
http://dergipark.gov.tr/asujse
http://asujse.aksaray.edu.tr

Research Article

\title{
New exact solution for $(2+1)$ and $(3+1)$ dimensional nonlinear partial differential equations
}

\author{
Ozkan Guner $^{*}$ \\ Department of International Trade, Cankiri Karatekin University, Cankiri 18100, Turkey
}

-Received Date: 10 May 2018

-Revised Date: 17 Oct 2018

-Accepted Date: 17 Oct 2018

-Published Online: 11 Dec 2018

\begin{abstract}
In this paper, dark soliton solutions have been obtained for the $(2+1)$-dimensional reactiondiffusion equation, the (3+1)-dimensional generalized B-type Kadomtsev-Petviashvili (gBKP) equation and the (3+1)-dimensional Boiti-Leon-Manna-Pempinelli (BLMP) equation using the solitary wave ansatz. Ansatz approach is utilized to carry out this integration. The constraint relations for each of the equations are given for the existence of dark soliton solutions.
\end{abstract}

\section{Keywords}

Ansatz method, dark soliton solutions, exact solution

\section{INTRODUCTION}

Nonlinear partial differential equations (NLPDEs) that are studied in the fields of physics and applied mathematics there are significant advances and across the globe many aspects of NLPDEs that are studied by various scientists. Some of them are the integrability aspects, symmetry issues, conservation laws. There has been quite thoroughly understood is the integrability aspect of the governing equations of the solitary waves in $(2+1)$-dimensional as well as in (3+1)-dimensional equations. For a long time, there are many new results related this aspects that are published in several area [1-6]. More commonly used methods of integrability are the He's variational iteration method, the hirota bilinear method, the homotopy perturbation

"Corresponding Author: Ozkan Guner, ozkanguner@karatekin.edu.tr 
method, the first integral method, the modified tanh--coth method, the modified sine-cosine method, the ansatz method and many others [7-12]. Among the methods mentioned above, the ansatz method is one of the most efficient method to determine solutions of NLPDEs [13-22]. It was originally developed by Anjan Biswas [23-25].

In nonlinear dynamical systems, propagation of nonlinear waves has been a fundamental objects of nature. Nonlinear waves emerge to in a great array of contexts such as, solid state physics, hydrodynamics, nuclear physics, plasmas, nonlinear optics and many other nonlinear phenomena. In this paper, we apply the ansatz method to obtain dark soliton solutions of the following (2+1)-dimensional reaction-diffusion equation, the $(3+1)$-dimensional $\mathrm{gBKP}$ equation and the (3+1)-dimensional BLMP equation. Dark soliton solutions is one of the fastest growing research fields in the context of wave phenomena [26, 27].

\section{THE (2+1)-DIMENSIONAL REACTION-DIFFUSION EQUATION}

Let us consider the following reaction-diffusion equation in two dimensions,

$$
\left(\psi_{\mathrm{t}}-\mathrm{D} \psi_{\mathrm{xx}}+\alpha \psi^{3}+\beta \psi^{2}+\gamma \psi\right)_{\mathrm{x}}+\mathrm{k} \psi_{\mathrm{yy}}=0
$$

where $\mathrm{D}, \alpha, \beta, \gamma$ and $\mathrm{k}$ are real constants, $\mathrm{D}$ is also called diffusion coefficient. In [28], YunQuan and Jun investigated the explicit analytical solutions for this equation (2.1) by using the first integral method.

For $\mathrm{k}=0$, equation (2.1) is a reaction-diffusion equation arising in chemical reaction or ecology and other fields of physics. The choice $\mathrm{D}=1, \beta=0$ and $\gamma=-\alpha$ leads Eq. (2.1) to the twodimensional Chaffee-Infante equation,

$$
\left(\psi_{\mathrm{t}}-\psi_{\mathrm{xx}}+\alpha \psi^{3}-\alpha \psi\right)_{\mathrm{x}}+\mathrm{k} \psi_{\mathrm{yy}}=0
$$

the choice $\mathrm{D}=1, \gamma=0$ and $\alpha=-\beta=1$ leads Eq. (2.1) to the two-dimensional Huxley equation,

$$
\left(\psi_{\mathrm{t}}-\psi_{\mathrm{xx}}+\psi^{3}-\psi\right)_{\mathrm{x}}+\mathrm{k} \psi_{\mathrm{yy}}=0
$$

and if taking $\mathrm{D}=1, \alpha=1, \beta=-(\mathrm{a}+1)$ and $\gamma=\mathrm{a}$ in Eq. (2.1), we can obtain the following twodimensional Fitzhugh--Nagumo equation:

$$
\left(\psi_{\mathrm{t}}-\psi_{\mathrm{xx}}+\psi^{3}-(\mathrm{a}+1) \psi^{2}+\mathrm{a} \psi\right)_{\mathrm{x}}+\mathrm{k} \psi_{\mathrm{yy}}=0 .
$$

In order to get its solution, the following ansatz is assumed [24, 29],

$$
\psi(\mathrm{x}, \mathrm{t})=\lambda \tanh ^{\mathrm{p}} \tau,
$$

and

$$
\tau=\mathrm{ax}+\mathrm{by}-\mathrm{vt},
$$

where the $\lambda$, $a$ and $b$ are the free parameters, and $v$ is the velocity of the soliton. From Eqs. (2.5) and (2.6), we have: 


$$
\begin{aligned}
& -\mathrm{p} \lambda \operatorname{va}\left\{(\mathrm{p}+1) \tanh ^{\mathrm{p}+2} \tau-2 \operatorname{ptanh}^{\mathrm{p}} \tau+(\mathrm{p}-1) \tanh ^{\mathrm{p}-2} \tau\right\} \\
& -\mathrm{D} \lambda \mathrm{pa}^{3}\left\{(\mathrm{p}-1)(\mathrm{p}-2)\left(\tanh ^{\mathrm{p}-3} \tau-\tanh ^{\mathrm{p}-1}\right\} \tau\right) \\
& \left.+(\mathrm{p}+1)(\mathrm{p}+2)\left(\tanh ^{\mathrm{p}+1} \tau-\tanh ^{\mathrm{p}+3} \tau\right)+2 \mathrm{p}^{2}\left(\tanh ^{\mathrm{p}+1} \tau-\tanh ^{\mathrm{p}-1} \tau\right)\right\} \\
& +3 \alpha \lambda^{3} \mathrm{pa}\left\{\tanh ^{3 \mathrm{p}-1} \tau-\tanh ^{3 \mathrm{p}+1} \tau\right\}+2 \beta \lambda^{2} \mathrm{pa}\left\{\tanh ^{2 \mathrm{p}-1} \tau-\tanh ^{2 \mathrm{p}+1} \tau\right\} \\
& +\gamma \lambda \mathrm{pa}\left\{\tanh ^{\mathrm{p}-1} \tau-\tanh ^{\mathrm{p}+1} \tau\right\}+\mathrm{k} \lambda \mathrm{pb}^{2}\left\{(\mathrm{p}-1) \tanh ^{\mathrm{p}-2} \tau-2 \operatorname{ptanh}^{\mathrm{p}} \tau+(\mathrm{p}+1) \tanh ^{\mathrm{p}+2} \tau\right\}=0 . \\
& \text { By equating the highest exponents of } \tanh ^{3 \mathrm{p}+1} \tau \text { and } \tanh ^{\mathrm{p}+3} \tau \text { terms in Eq }(2.7), \text { we }
\end{aligned}
$$

obtain

$$
\begin{gathered}
3 p+1=p+3, \\
p=1 .
\end{gathered}
$$

The same value of $p$ can yield when the exponents pairs $2 p+1$ and $p+2,2 p-1$ and $p$ are equate with each other. Furthermore, set the coefficients of the linearly independent terms to zero. By setting the corresponding coefficients of $\tanh ^{3 \mathrm{p}+1} \tau$ and $\tanh ^{\mathrm{p}+3} \tau$ terms to zero we get

$$
D \lambda p(p+1)(p+2) a^{3}-3 \alpha \lambda^{3} p a=0,
$$

after some calculations we obtain

$$
\lambda= \pm \mathrm{a} \sqrt{\frac{(\mathrm{p}+1)(\mathrm{p}+2) \mathrm{D}}{3 \alpha}},
$$

which implies that is necessary to have $\mathrm{D} \alpha>0$.

Setting the coefficient of $\tanh ^{\mathrm{p}-1} \tau$ terms in Eq. (2.7), to one obtains

$$
2 \mathrm{p}^{3} \mathrm{D} \lambda \mathrm{a}^{3}+\gamma \lambda \mathrm{pa}=0,
$$

which gives

$$
\mathrm{a}= \pm \sqrt{\frac{\gamma}{2 \mathrm{p}^{2} \mathrm{D}}} .
$$

Thus from (2.12) it is possible to conclude that the topological solitons will exist for $\gamma \mathrm{D}<0$. Again from (2.7), matching the exponents of $\tanh ^{2 \mathrm{p}+1} \tau$ and $\tanh ^{\mathrm{p}+2} \tau$ to zero yields

$$
-p(p+1) \lambda v a-2 \beta \lambda^{2} p a+k \lambda p(p+1) b^{2}=0,
$$

we obtain

$$
\mathrm{v}=\frac{2 \beta \lambda \mathrm{a}-\mathrm{k}(\mathrm{p}+1) \mathrm{b}^{2}}{(\mathrm{p}+1) \mathrm{a}} .
$$

Hence, we obtain the topological soliton solution for the equation (2.1) when we substitude (2.8), (2.10), (2.12), (2.14) in (2.5) as

$$
\psi(x, y, t)=\lambda \tanh (a x+b y-v t),
$$

which exist provided that $\gamma \mathrm{D}<0$ and $\mathrm{D} \alpha>0$. 


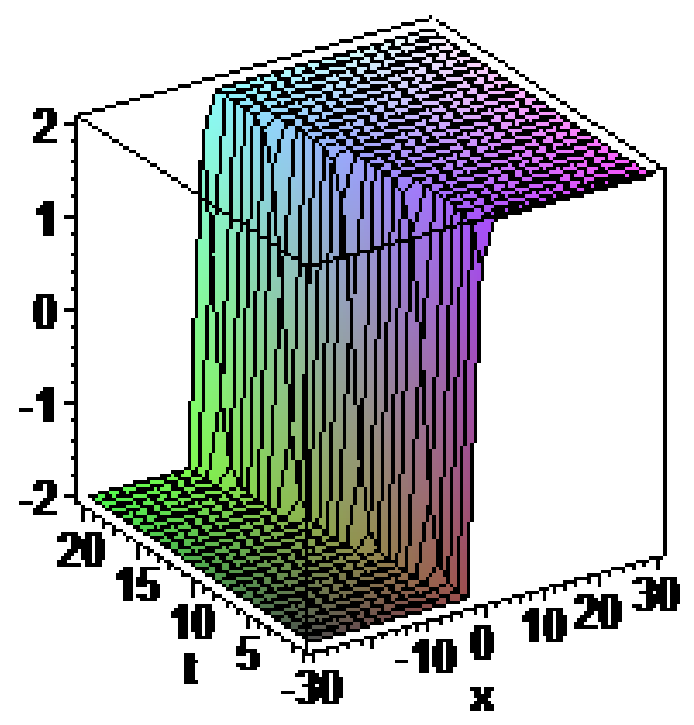

Figure 1. Shock wave $\psi(\mathrm{x}, 1, \mathrm{t})$ with $\gamma=-8, \alpha=2, \beta=1, \mathrm{k}=2, \mathrm{D}=1, \mathrm{~b}=1$.

\section{THE (3+1)-DIMENSIONAL GENERALIZED B-TYPE KADOMTSEV PETVIASHVILI (gBKP) EQUATION}

Now we consider the (3+1)-dimensional gBKP equation [30],

$$
\psi_{\mathrm{xxxy}}+\alpha\left(\psi_{\mathrm{x}} \psi_{\mathrm{y}}\right)_{\mathrm{x}}+\beta\left(\psi_{\mathrm{x}}+\psi_{\mathrm{y}}+\psi_{\mathrm{z}}\right)_{\mathrm{t}}+\gamma\left(\psi_{\mathrm{xx}}+\psi_{\mathrm{zz}}\right)=0,
$$

where $\alpha, \beta$ and $\gamma$ are real-valued constant. This is a nonlinear wave equation in three spatial $(\mathrm{x}, \mathrm{y}, \mathrm{z})$ and one temporal coordinate $(\mathrm{t})$. Khalique and Abudiab studied the generalized BKP equation and derived some exact solutions using the multiple-exp function and simplest equation methods. Also, the conservation laws for the BKP equation are constructed by using the multiplier method by them. In [31], a new form of the (3+1)-dimensional BKP equation given by (3.1) was investigated and it was shown, using the simplified form of the Hirota method, that one- and two-soliton solutions exist for (3.1). Also, specific constraints were developed that guarantee the existence of multiple soliton solutions for $(3.1)$. The $(3+1)$ dimensional nonlinear gBKP equation

$$
\psi_{\mathrm{yt}}-\psi_{\mathrm{xxxy}}-3\left(\psi_{\mathrm{x}} \psi_{\mathrm{y}}\right)_{\mathrm{x}}+3\left(\psi_{\mathrm{xx}}+\psi_{\mathrm{zz}}\right)=0
$$

was studied in [32-35] by different approaches.

The other variant of a generalized (3+1)-dimensional BKP equation given by

$$
\psi_{\mathrm{xxxy}}-3\left(\psi_{\mathrm{x}} \psi_{\mathrm{y}}\right)_{\mathrm{x}}+\psi_{\mathrm{tx}}+\psi_{\mathrm{ty}}-\psi_{\mathrm{zz}}=0
$$

In [36], Ma and Abdeljabbar has computed a bilinear Bäcklund transformation for Eq. (3.3) and in [37], Wronskian and Grammian formulations are established for this equation. Ma and Zhu 
[38], applied the multiple exp-function algorithm to this equation. In [39], Wazwaz studied three variants of a generalized (3+1)-dimensional BKP equation in the form

$$
\begin{aligned}
& \psi_{\mathrm{zt}}-\psi_{\mathrm{xxxy}}-3\left(\psi_{\mathrm{x}} \psi_{\mathrm{y}}\right)_{\mathrm{x}}+3 \psi_{\mathrm{xx}}=0, \\
& \psi_{\mathrm{yt}}-\psi_{\mathrm{xxxz}}-3\left(\psi_{\mathrm{x}} \psi_{\mathrm{z}}\right)_{\mathrm{x}}+3 \psi_{\mathrm{yy}}=0, \\
& \psi_{\mathrm{xt}}-\psi_{\mathrm{xxxy}}-3\left(\psi_{\mathrm{x}} \psi_{\mathrm{y}}\right)_{\mathrm{x}}+3 \psi_{\mathrm{zz}}=0,
\end{aligned}
$$

and multiple-front wave solutions of Eq. (3.4) have been obtained by using the simplified form of the Hirota's method. Also, in [40] Ma and Fan studied the first (3+1)-dimensional gBKP equation and presenting its particular exact multiple wave solutions.

In order to construct dark soliton solutions, we use

$$
\psi(\mathrm{x}, \mathrm{y}, \mathrm{z}, \mathrm{t})=\lambda \tanh ^{\mathrm{p}} \tau
$$

and

$$
\tau=\mathrm{ax}+\mathrm{by}+\mathrm{cz}-\mathrm{vt},
$$

where $\lambda, a, b$ and $c$ are free parameters and $v$ is the velocity of the soliton. From Eq. (3.5) and (3.6), we have

$$
\begin{aligned}
& \lambda \mathrm{pa}^{3} \mathrm{~b}\left\{(\mathrm{p}-1)(\mathrm{p}-2)(\mathrm{p}-3) \tanh ^{\mathrm{p}-4} \tau-4(\mathrm{p}-1)\left(\mathrm{p}^{2}-2 \mathrm{p}+2\right) \tanh ^{\mathrm{p}-2} \tau+2 \mathrm{p}\left(3 \mathrm{p}^{2}+5\right) \tanh ^{\mathrm{p}} \tau\right. \\
& \left.-4(\mathrm{p}+1)\left(\mathrm{p}^{2}+2 \mathrm{p}+2\right) \tanh ^{\mathrm{p}+2} \tau+(\mathrm{p}+1)(\mathrm{p}+2)(\mathrm{p}+3) \tanh ^{\mathrm{p}+4} \tau\right\}+\alpha \lambda^{2} \mathrm{p}^{2} \mathrm{a}^{2} \mathrm{~b}\left\{(2 \mathrm{p}-2) \tanh ^{\wedge}\{2 \mathrm{p}-3\} \tau\right. \\
& \left.-(6 \mathrm{p}-2) \tanh ^{2 \mathrm{p}-1} \tau+(6 \mathrm{p}+2) \tanh ^{2 \mathrm{p}+1} \tau-(2 \mathrm{p}+2) \tanh ^{2 \mathrm{p}+3} \tau\right\} \\
& -\beta \mathrm{p} \lambda \mathrm{v}(\mathrm{a}+\mathrm{b}+\mathrm{c})\left\{(\mathrm{p}+1) \tanh ^{\mathrm{p}+2} \tau-2 \operatorname{ptanh}^{\mathrm{p}} \tau+(\mathrm{p}-1) \tanh ^{\mathrm{p}-2} \tau\right\} \\
& +\gamma \mathrm{p} \lambda\left(\mathrm{a}^{2}+\mathrm{c}^{2}\right)\left\{(\mathrm{p}-1) \tanh ^{\mathrm{p}-2} \tau-2 \operatorname{ptanh}^{\mathrm{p}} \tau+(\mathrm{p}+1) \tanh ^{\mathrm{p}+2} \tau\right\}=0 .
\end{aligned}
$$

When we match the exponents of $\tanh ^{2 \mathrm{p}+3} \tau$ and $\tanh ^{\mathrm{p}+4} \tau$ terms in Eq (3.7), we obtain

$$
\begin{aligned}
2 p+3 & =p+4, \\
p & =1 .
\end{aligned}
$$

By setting the coefficients of $\tanh ^{2 \mathrm{p}+3} \tau$ and $\tanh ^{\mathrm{p}+4} \tau$ terms to zero we get

$$
\lambda p(p+1)(p+2)(p+3) a^{3} b-2 \alpha \lambda^{2} p^{2}(p+1) a^{2} b=0,
$$

so that

$$
\lambda=\frac{(\mathrm{p}+2)(\mathrm{p}+3) \mathrm{a}}{2 \alpha \mathrm{p}}
$$

Again, by equating the coefficients of $\tanh ^{2 \mathrm{p}+1} \tau$ and $\tanh ^{\mathrm{p}+2} \tau$ terms to zero

$$
-4 \lambda \mathrm{pa}^{3} b(p+1)\left(p^{2}+2 p+2\right)+\alpha \lambda^{2} p^{2}(6 p+2) a^{2} b-\beta p(p+1) \lambda v(a+b+c)+\gamma p(p+1) \lambda\left(a^{2}+c^{2}\right)=0 .
$$


Solving the equation (3.12) with by using (3.11)

$$
\mathrm{V}=\frac{-4(p+1)\left(p^{2}+2 p+2\right) a^{3} b+\alpha(3 p+1)(p+2)(p+3) a^{3} b+\gamma(p+1)\left(a^{2}+c^{2}\right)}{p(p+1) \beta(a+b+c)} .
$$

The relations (3.11) and (3.13) introduce the restrictions given by

$$
\begin{array}{r}
\alpha \neq 0, \\
\beta(a+b+c) \neq 0 .
\end{array}
$$

Dark soliton solution for the equation (3.1) is given by

$$
\psi(\mathrm{x}, \mathrm{y}, \mathrm{z}, \mathrm{t})=\lambda \tanh (\mathrm{ax}+\mathrm{by}+\mathrm{cz}-\mathrm{vt})
$$

where the free parameter $\lambda$ is given by (3.11) and velocity of the solitons $v$ is given in (3.13). In (3.13) $\mathrm{v}$ is dependent on the other free parameters $\mathrm{a}, \mathrm{b}$ and $\mathrm{c}$.

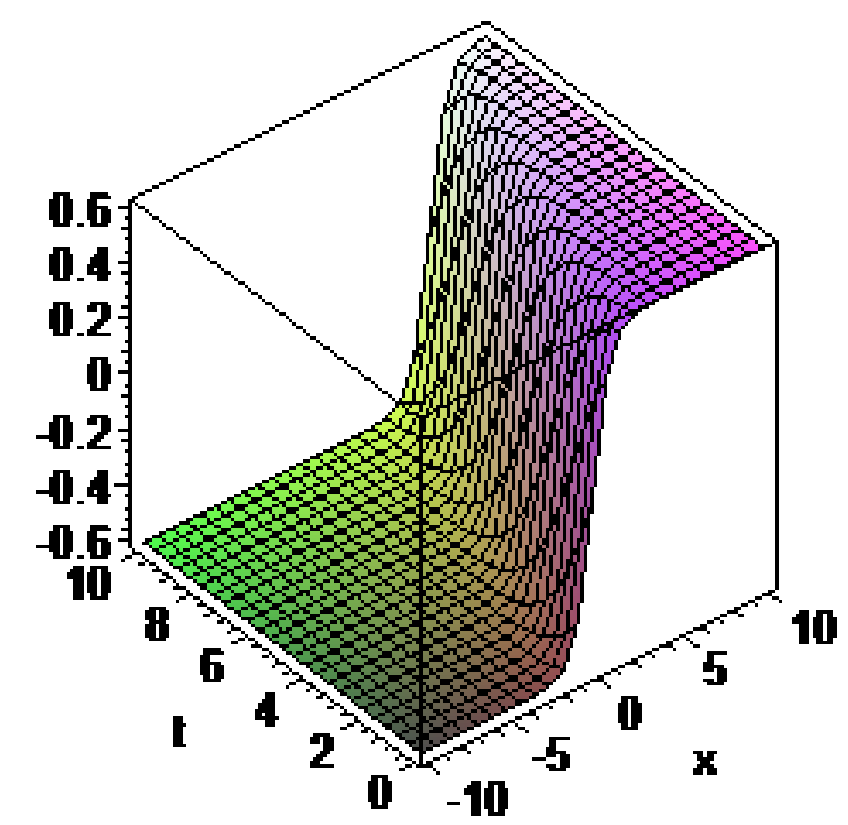

Figure 2. Shock wave Eq.(3.1) with $\gamma=1, \alpha=10, \beta=10, a=1, b=10, c=10, p=1$

\section{THE (3+1)-DIMENSIONAL BOITI-LEON-MANNA-PEMPINELLI EQUATION}

For initial data decaying rapidly at infinity, Boiti, Leon, Manna and Pempinelli have developed an inverse scattering scheme to solve the Cauchy problem of a (2+1)-dimensional generalization of the shallow water wave equations:

$$
\psi_{\mathrm{yt}}+\psi_{\mathrm{xxxy}}-3 \psi_{\mathrm{xx}} \psi_{\mathrm{y}}-3 \psi_{\mathrm{x}} \psi_{\mathrm{xy}}=0
$$

Eq. (4.1), it is also known (2+1)-dimensional BLMP equation has been studied in detail by many scientists. For example, Song-Hua and Jian-Ping obtained various exact solutions which 
include solitary wave solution and by selecting appropriate functions, they investigated some novel localized excitations such as multi dromion-solitoffs and fractal-solitons. Eq. (4.1) has been considered as a (2+1)-dimensional generalization of the shallow water wave equations in [41]. Painlevé analysis, Lax pair and some exact solutions of Eq. (4.1) have been given in [42] and some soliton-like solutions have been obtained through the symbolic-computation-based method in [43]. Gao an Tian studied this equation and derived some new soliton-like solutions using the symbolic computation with the generalized tanh method [44].

Now, we introduce an extension to Eq. (4.1) by adding the last three terms with y replaced by z. This extension enables us to establish a BLMP equation as

$$
\psi_{\mathrm{yt}}+\psi_{\mathrm{zt}}+\psi_{\mathrm{xxxy}}+\psi_{\mathrm{xxxz}}-3 \psi_{\mathrm{x}}\left(\psi_{\mathrm{xy}}+\psi_{\mathrm{xz}}\right)-3 \psi_{\mathrm{xx}} \psi_{\mathrm{y}}-3 \psi_{\mathrm{xx}} \psi_{\mathrm{z}}=0,
$$

In [45], Darvishi et al. have obtained single-wave, double-wave and multi-wave solutions of Eq. (4.2) by the multiple exp-function method. Similarly, in order to construct dark soliton solutions for Eq. (4.2), we use an ansatz solution of the form (3.5) and (3.6). From Eq. (3.5) and (3.6), we have

$-\mathrm{p} \lambda \mathrm{v}(\mathrm{b}+\mathrm{c})\left\{(\mathrm{p}+1) \tanh ^{\mathrm{p}+2} \tau-2 \operatorname{ptanh}^{\mathrm{p}} \tau+(\mathrm{p}-1) \tanh ^{\mathrm{p}-2} \tau\right\}+\lambda \mathrm{pa}^{3}(\mathrm{~b}+\mathrm{c})\left\{(\mathrm{p}-1)(\mathrm{p}-2)(\mathrm{p}-3) \tanh ^{\mathrm{p}-4} \tau\right.$

$-4(\mathrm{p}-1)\left(\mathrm{p}^{2}-2 \mathrm{p}+2\right) \tanh ^{\mathrm{p}-2} \tau+2 \mathrm{p}\left(3 \mathrm{p}^{2}+5\right) \tanh ^{\mathrm{p}} \tau-4(\mathrm{p}+1)\left(\mathrm{p}^{2}+2 \mathrm{p}+2\right) \tanh ^{\mathrm{p}+2} \tau$

$\left.+(\mathrm{p}+1)(\mathrm{p}+2)(\mathrm{p}+3) \tanh ^{\mathrm{p}+4} \tau\right\}-6 \lambda^{2} \mathrm{p}^{2} \mathrm{a}^{2}(\mathrm{~b}+\mathrm{c})\left\{(\mathrm{p}-1) \tanh ^{2 \mathrm{p}-3} \tau-(3 \mathrm{p}-1) \tanh ^{2 \mathrm{p}-1} \tau\right.$

$\left.+(3 \mathrm{p}+1) \tanh ^{2 \mathrm{p}+1} \tau-(\mathrm{p}+1) \tanh ^{2 \mathrm{p}+3} \tau\right\}=0$.

By equating the exponents of $\tanh ^{2 \mathrm{p}+3} \tau$ and $\tanh ^{\mathrm{p}+4} \tau$ terms in $\mathrm{Eq}(4.3)$,

$$
2 p+3=p+4
$$

we get

$$
\mathrm{p}=1
$$

Furthermore, set the coefficients of the linearly independent terms to zero.

$$
\begin{gathered}
\lambda p(p+1)(p+2)(p+3) a^{3}(b+c)+6 \lambda^{2} p^{2}(p+1) a^{2}(b+c)=0, \\
-p(p+1) \lambda v(b+c)-4 \lambda a^{3}(b+c) p(p+1)\left(p^{2}+2 p+2\right)-6 \lambda^{2} p^{2}(3 p+1) a^{2}(b+c)=0 .
\end{gathered}
$$

Solving the above system of equations and also set $p=1$, then it can be written

$$
\begin{gathered}
\lambda=-2 \mathrm{a}, \\
\mathrm{v}=4 \mathrm{a}^{3} .
\end{gathered}
$$


Further, the Eqs. (4.6) and (4.7) imply that the solution will exist for $b+c \neq 0$. Therefore the dark soliton solution or topological 1-soliton solution of (3+1)-dimensional BLMP equation (4.2) is given by

$$
\psi(\mathrm{x}, \mathrm{y}, \mathrm{z}, \mathrm{t})=-2 \mathrm{a} \cdot \tanh \left(\mathrm{ax}+\mathrm{by}+\mathrm{cz}-4 \mathrm{a}^{3} \mathrm{t}\right),
$$

Remark: Solution (4.10) is also solution of the (2+1)-dimensional BLMP equation.

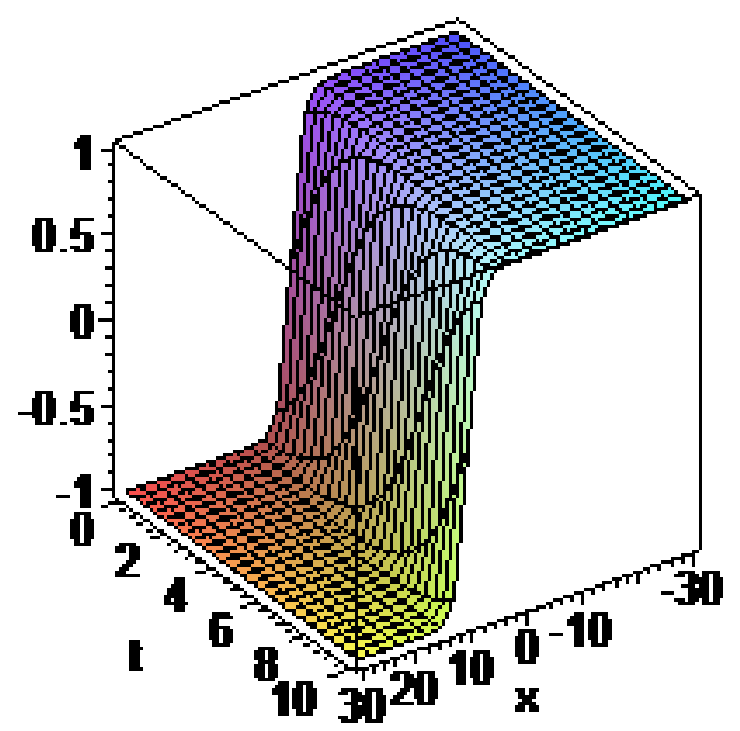

Figure 3. Dark soliton solution Eq.(4.2) with $\mathrm{a}=1, \mathrm{p}=1$

\section{CONCLUSION}

In this paper; dark soliton solutions of the $(2+1)$-dimensional reaction-diffusion, the $(3+1)$ dimensional generalized B-type Kadomtsev-Petviashvili (BKP) and the (3+1)-dimensional BLMP equations are obtained by ansatz method. The ansatz method was employed to carry out the integration. The dark soliton solutions also introduced several constraint conditions that must remain valid in order for these solutions to exist.

\section{References}

[1] M. Antonova, A. Biswas, Adiabatic parameter dynamics of perturbed solitary waves. Commun. Nonlinear Sci. Numer. Simulat. 14 (2009) 734-748.

[2] L., Zhang, Convergence of a conservative difference scheme for a class of Klein-GordonSchrödinger equations in one space dimension. Appl. Math. Comput. 163 (2005) 343-355.

[3] A.M., Wazwaz, New solitary wave solutions to the modified Kawahara equation. Phys. Lett. A 360 (2007) 588-592.

[4] Z., Yang, W.P., Zhong, Analytical solutions to Sine-Gordon equation with variable coefficient. Romanian Reports in Physics 66 (2014) 262-273.

[5] D.M., Mothibi, C.M., Khalique, On the exact solutions of a modified Kortweg de Vries type equation and higher-order modified Boussinesq equation with damping term. Adv. Differ. Equ. 2013 (2013) 166. 
[6] U.M., Abdelsalam, Traveling wave solutions for shallow water equations. Journal of Ocean Engineering and Science 2 (2017) 28-33.

[7] C., Cattani, T.A., Sulaiman, H.M., Baskonus, H., Bulut, On the soliton solutions to the NizhnikNovikov-Veselov and the Drinfel'd-Sokolov systems. Opt Quant Electron (2018) 50:138.

[8] C., Cattani, T.A., Sulaiman, H.M., Baskonus, H., Bulut, Solitons in an inhomogeneous Murnaghan's rod. Eur. Phys. J. Plus (2018) 133: 228.

[9] H.M., Baskonus, New acoustic wave behaviors to the Davey--Stewartson equation with power-law nonlinearity arising in fluid Dynamics. Nonlinear Dyn. 86 (2016) 177-183.

[10] H.M.,Baskonus, New complex and hyperbolic function solutions to the generalized double combined Sinh-Cosh-Gordon equation. AIP Conference Proceedings 1798 (2017) 020018.

[11] H.M., Baskonus, H., Bulut New Hyperbolic Function Solutions for Some Nonlinear Partial Differential Equation Arising in Mathematical Physics. Entropy 17 (2015) 4255-4270.

[12] H.M., Baskonus, H., Bulut An Effective Schema for Solving Some Nonlinear Partial Differential Equation Arising In Nonlinear Physics. Open Phys. 13 (2015) 280-289.

[13] J., Biazara, H., Ghazvini, He's variational iteration method for solving linear and non-linear systems of ordinary differential equations. Appl. Math. Comput. 191 (2007) 287-297.

[14] E., Babolian, A., Azizi, J., Saeidian, Some notes on using the homotopy perturbation method for solving time-dependent differential equations. Mathematical and Computer Modelling 50 (2009) 213-224.

[15] Z., Jin-Ming, Z., Yao-Ming, The Hirota bilinear method for the coupled Burgers equation and the high-order Boussinesq Burgers equation. Chin. Phys. B 20 (2011) 010205.

[16] S.A., El-Wakil, M.A., Abdou, New exact travelling wave solutions using modified extended tanhfunction method. Chaos, Solitons \& Fractals 31 (2007) 840-852.

[17] Guner, O., Bekir, A., Traveling wave solutions for time-dependent coefficient nonlinear evolution equations, Waves in Random and Complex Media, 253 (2015) 342-349

[18] M.S., Ismail, Numerical solution of complex modified Korteweg-de Vries equation by PetrovGalerkin method. Appl. Math. Comput. 202 (2008) 520-531.

[19] S., Kutluay, A., Esen, Exp-function method for solving the general improved KdV equation. International Journal of Nonlinear Sciences and Numerical Simulation 10 (2009) 717-725.

[20] A., Bekir, Application of the $\left(G^{\prime} / G\right)$-expansion method for nonlinear evolution equations. Phys. Lett. A 372 (2008) 3400-3406.

[21] N., Taghizadeh, M., Mirzazadeh, The first integral method to some complex nonlinear partial differential equations. Journal of Computational and Applied Mathematics 235 (2011) 4871-4877.

[22] A., Bekir, O., Guner, Bright and dark soliton solutions of the $(3+1)$-dimensional generalized Kadomtsev--Petviashvili equation and generalized Benjamin equation. Pramana - J. Phys. 81 (2013) 203-214.

[23] A., Biswas, 1-Soliton solution of the $K(m, n)$ equation with generalized evolution. Phys. Lett. A 372 (2008a) 4601-4602.

[24] A., Biswas, 1-Soliton solution of $(1+2)$ dimensional nonlinear Schrödinger's equation in dualpower law media. Phys. Lett. A 372 (2008b) 5941-5943.

[25] A., Biswas, M.D., Petkovic', D., Milovic, Topological and non-topological exact soliton solution of the power law KdV equation. Commun Nonlinear Sci Numer Simulat. 15 (2010) 3263-3269.

[26] A., Biswas, D., Milovic, Bright and dark solitons of the generalized nonlinear Schrödinger's equation. Commun. Nonlinear Sci. Numer. Simulat. 15 (2010) 1473-1484. 
[27] M., Saha, A.K., Sarma, A., Biswas, Dark optical solitons in power law media with time-dependent coefficients. Phys. Lett. A 373 (2009) 4438-4441.

[28] K., Yun-Quan, Y., Jun, The First Integral Method to Study a Class of Reaction-Diffusion Equations. Commun. Theor. Phys. 43 (2005) 597-600.

[29] A., Bekir, O., Guner, Topological (dark) soliton solutions for the Camassa--Holm type equations. Ocean Engineering 74 (2013) 276-279.

[30] M., Abudiab, C.M., Khalique, Exact solutions and conservation laws of a $(3+1)$-dimensional Btype Kadomtsev-Petviashvili equation. Advances in Difference Equations 2013 (2013):221.

[31] A.M., Wazwaz, Two forms of (3+1)-dimensional B-type Kadomtsev-Petviashvili equation: multiple-soliton solutions. Phys. Scr. 86 (2012) 035007.

[32] A.M., Wazwaz, Distinct kinds of multiple-soliton solutions for a $(3+1)$-dimensional generalized B-type Kadomtsev-Petviashvili equation. Phys. Scr. 84 (2011) 055006.

[33] H.F., Shen, M.H., Tu, On the constrained B-type Kadomtsev-Petviashvili equation: Hirota bilinear equations and Virasoro symmetry. J. Math. Phys. 52 (2011) 032704.

[34] W.X., Ma, Y., Zhang, Y., Tang, J., Tu, Hirota bilinear equations with linear subspaces of solutions. Appl. Math. Comput. 218 (2012) 7174-7183.

[35] M.G., Asaad, W.X., Ma, Pfaffian solutions to a (3+1)-dimensional generalized B-type Kadomtsev-Petviashvili equation and its modified counterpart. Appl. Math. Comput. 218 (2012) 5524-5542.

[36] W.X., Ma, A., Abdeljabbar, A bilinear Bäcklund transformation of a $(3+1)$-dimensional generalized KP equation. Appl. Math. Lett. 25 (2012) 1500-1504.

[37] W.X., Ma, A., Abdeljabbar, M.G., Asaad, Wronskian and Grammian solutions to a $(3+1)$ dimensional generalized KP equation. Appl. Math. Comput. 217 (2011) 10016-10023.

[38] W.X., Ma, Z., Zhu, Solving the $(3+1)$-dimensional generalized KP and BKP equations by the multiple exp-function algorithm. Appl. Math. Comput. 218 (2012) 11871-11879.

[39] A.M., Wazwaz, Variants of a (3+1)-dimensional generalized BKP equation:Multiple-front waves solutions. Computers \& Fluids 97 (2014) 164-167.

[40] W.X., Ma, E., Fan, Linear superposition principle applying to Hirota bilinear equations, Computers and Mathematics with Applications. 61 (2011) 950-959.

[41] M., Boiti, J.J. -P., Leon, M., Manna, F., Pempinelli, On the spectral transform of a Korteweg-de Vries equation in two spatial dimensions. Inverse Problems 2 (1986) 271-279.

[42] P.G., Estévez, S.B., Leble, A Wave Equation in 2+1: Painlevé Analysis and Solutions. Inverse Problems 11 (1995) 925-937.

[43] B., Tian, Y.T., Gao, Soliton-like solutions for a $(2+1)$-dimensional generalization of the shallow water wave equations. Chaos, Solitons \& Fractals 7 (1996) 1497-1499.

[44] Y.T., Gao, B., Tian, Generalized Tanh Method with Symbolic Computation and Generalized Shallow Water Wave Equation. Computers Math. Applic. 33 (1997) 115-118.

[45] M.T., Darvishi, M., Najafi, L., Kavitha, M., Venkatesh, Stair and Step Soliton Solutions of the Integrable $(2+1)$ and $(3+1)$-Dimensional Boiti-Leon--Manna--Pempinelli Equations. Commun. Theor. Phys. 58 (2012) 785-794. 